\title{
3-氨基-1,2,4-三氮唑对铜的缓蚀性能和吸附行为
}

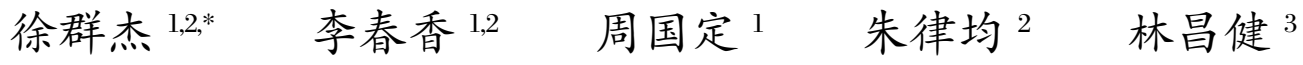 \\ ( ${ }^{1}$ 上海电力学院环境工程系, 上海 200090； ${ }^{2}$ 上海大学应用化学系, 上海 200444; \\ ${ }^{3}$ 厦门大学固体表面物理化学国家重点实验室, 福建 厦门 361005)
}

\begin{abstract}
摘要: 采用电化学和表面增强拉曼光谱(SERS)方法研究了新型环境友好型金属水处理剂 3-氨基-1,2,4-三氮唑 (ATA)对铜在 3\% $\mathrm{NaCl}$ 溶液中的缓蚀性能和吸附行为. 结果表明, ATA 对铜有较好的缓蚀作用, 其中 ATA 浓度 为 $0.24 \mathrm{mmol} \cdot \mathrm{L}^{-1}$ 时对铜的缓蚀效率最高, 为 $97.65 \%$, 其吸附行为符合 Langmuir 吸附等温式, 吸附机理是典型 的化学吸附. SERS 表明, ATA 分子通过很强的吸附于铜表面达到抑制其腐蚀的作用, 是 ATA-与 $\mathrm{Cu}^{+}$形成配合物 来阻止氯离子化合物 $\left(\mathrm{CuCl}_{2}\right)$ 的生成.
\end{abstract}

关键词：铜; ATA; 缓蚀剂 电化学; SERS

中图分类号: $\mathrm{O} 646$

\section{Copper Corrosion Inhibition and Adsorption Behavior of 3-Amino-1,2,4-triazole}

\author{
XU Qun-Jie ${ }^{1,2, *} \quad$ LI Chun-Xiang ${ }^{1,2} \quad$ ZHOU Guo-Ding ${ }^{1} \quad$ ZHU Lü-Jun $^{2} \quad$ LIN Chang-Jian ${ }^{3}$ \\ ('Department of Environment Engieering, Shanghai University of Electric Power, Shanghai 200090, P. R. China; ${ }^{2}$ Department of \\ Chemical Engineering, Shanghai University, Shanghai 200444, P. R. China; ${ }^{3}$ State Key Laboratory of Solid Surface Physical \\ Chemistry, Xiamen University, Xiamen 361005, Fujian Province, P. R. China)
}

\begin{abstract}
Corrosion inhibition of copper in $3 \% \mathrm{NaCl}$ solution by 3-amino-1,2,4-triazole (ATA) was studied in relation to the concentration of the inhibitor using electrochemical (ac impedance and dc polarization) and surface enhanced Raman spectroscopy (SERS) techniques. The results indicated that ATA was a good corrosion inhibiter for copper in a 3\% $\mathrm{NaCl}$ solution. The inhibition efficiency was $97.65 \%$ at an ATA concentration of $20 \mathrm{mg} \cdot \mathrm{L}^{-1}$. Polarization curves showed that ATA behaved as a type of cathodical inhibitor in $3 \% \mathrm{NaCl}$ solution. Adsorption of ATA followed Langmuir's adsorption isotherm and the adsorption mechanism was typical of chemisorption. SERS revealed that inhibition of copper corrosion was due to adsorption of ATA molecules on the surface of copper. SERS also confirmed that the adsorbed ATA molecules formed a complex with $\mathrm{Cu}^{+}$which prevented the formation of copper chloride complexes, $\mathrm{CuCl}_{2}^{-}$.
\end{abstract}

Key Words: Copper; ATA; Inhibitor; Electrochemistry; SERS

铜因具有稳定的物理化学性质、优良的导热性 能及耐腐蚀能力而有被广泛地应用于建筑、电气、造 船、石油化工等许多重要工业领域. 但是, 在中性介 质中, 尤其是 $\mathrm{Cl}^{-}$等侵蚀性离子存在时 ${ }^{[1]}$, 铜腐蚀将
更为严重, 甚至会给人类的生产和生活带来极大的 危害. 投加缓蚀剂是控制金属腐蚀最经济的方法之 一, 所以人们不断深人研究缓蚀剂的作用 ${ }^{[2-8]}$. 目前 研究的缓蚀剂主要是含有 $\mathrm{N} 、 \mathrm{~S} 、 \mathrm{O}$ 或含有其它功能

Received: July 4, 2008; Revised: September 19, 2008; Published on Web: October 30, 2008.

*Corresponding author. Email: xuqunjie@ shiep.edu.cn; Tel: +8621-65430410-247.

国家自然科学基金(20406009)、上海市教委重点项目(06ZZ67)、上海市科技攻关计划(062312045)、上海市科委项目(08DZ2201400、

08DZ2210800)厦门大学固体表面物理化学国家重点实验室开放课题(200512)及上海市重点学科(P1304)资助

C. Editorial office of Acta Physico-Chimica Sinica 
官能团的有机物, 如苯并三氮唑(简称 BTA)已在 工业中应用数年. 然而, 这类缓蚀剂的主要缺点是 有毒, 而且其中的大多数不可生物降解. 因此, 新型 环境友好型缓蚀剂备受关注. 3-氨基-1,2,4-三氮唑 (简称 ATA) 属唑类有机物, 为环境友好型, 但作为缓 蚀剂研究甚少. 研究表明, ATA 在磷酸氢钾与盐酸 的混合液中对铜腐蚀有一定的缓蚀作用 ${ }^{[0]}$, El-Sayed 等 ${ }^{[10]}$ 研究了在 $0.5 \mathrm{~mol} \cdot \mathrm{L}^{-1}$ 的 $\mathrm{HCl}$ 溶液中 ATA 对铜 腐蚀有较好的缓蚀作用.

3-氨基-1,2,4-三氮唑(结构式见图 1)是一种用途 广泛的有机合成中间体, 也是用于人体蛋白质中色 氨酸含量的特种生化试剂. 它具有很强的螯合性、光 敏性以及生物活性, 被广泛用于抗菌素类药物、三唑 类偶氨染料、感光材料、内吸性杀菌剂以及植物生长 调节剂的合成与制备. 此外, 它还可以直接用作除草 剂、润滑剂、金属缓蚀剂等. ATA 在缓蚀剂方面的研 究比较晚 ${ }^{[11]}$. 本文主要利用交流阻抗和极化曲线法 研究了 ATA 在 $3 \% \mathrm{NaCl}$ 溶液中对铜的缓蚀行为和 吸附行为. 同时, 本文在前期工作基础上通过表面增 强拉曼光谱的测试, 从分子水平上直接提供表面分 子结构和动态过程等重要信息, 研究了 ATA 的缓 蚀作用机理。

\section{1 实验部分}

\section{1 材料与试剂}

铜电极由纯铜(99.99\%)制得. 实验试剂均为分 析纯, 溶液由去离子水配制。

\section{2 测试方法}

交流阻抗和极化曲线的测试采用经典的三电极 体系, 工作电极为铜电极. 交流阻抗和极化曲线的测 定用 PARC M283 恒电位仪, PARC1025 频谱分析 仪, 配套软件为 PARCM398, PARCM352, 交流阻抗 的测量频率范围为 $100 \mathrm{kHz}-50 \mathrm{mHz}$, 交流激励信号 峰值为 $5 \mathrm{mV}$; 极化曲线的扫描速率为 $2 \mathrm{mV} \cdot \mathrm{s}^{-1}$.

现场表面增强拉曼光谱(SERS)测量在法国 Dilor 公司的 LABRAM I 型共焦显微拉曼光谱仪上进 行, 激光经显微镜镜头后垂直人射到研究电极表 面, 散射光经该物镜头背散射形式收集后再送到 拉曼谱仪. 激光波长为 $632.8 \mathrm{~nm}$, 由 $\mathrm{He}-\mathrm{Ne}$ 激光器 产生, 到达样品的最大功率不超过 $12 \mathrm{~mW}$. 用 EG\&GPARC173 恒电位仪控制电位, 本测试采用三 电极体系, 工作电极为纯铜, 直径 $2 \mathrm{~mm}$ 的圆铜片嵌 人比其外经略小的热聚四氟乙烯套中, 非工作面用

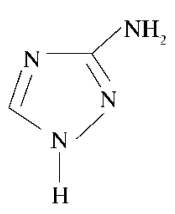

图 13 -氨基-1,2,4-三氮唑(ATA)的结构示意图

Fig.1 Structure of 3-amino-1,2,4-triazole (ATA) 环氧树酯封装.

实验中所用辅助电极均为铂电极, 参比电极均 为饱和甘录电极 (SCE), 本文所示电位均相对于饱和 甘采电极。

\section{3 电极的前处理}

在交流阻抗和极化曲线的测试中, 铜电极用 环氧树酯密封制成, 工作面积为 $0.24 \mathrm{~cm}^{2}$, 测量前铜 电极用金相砂纸逐级打磨抛光, 然后用无水乙醇进 行除油, 最后用去离子水冲洗干净后放人电解液中 于- $1.10 \mathrm{~V}$ 电位下阴极极化 $2 \mathrm{~min}$, 经去离子水冲洗 后于被测溶液中稳定 $30 \mathrm{~min}$.

测量前工作电极表面抛光至镜面, 然后超声清 洗, 最后用超纯水冲洗后移人电解池. 电解液为 0.1 $\mathrm{mol} \cdot \mathrm{L}^{-1} \mathrm{KCl}$, 控制电位在 $-0.4 \mathrm{~V}$ 到 $0.4 \mathrm{~V}$ 之间阶跃 多次即可得到较好的 SERS 活性.

\section{2 结果与讨论}

\section{1 不同浓度缓蚀剂下交流阻抗的测量}

图 2 为铜电极在未加和添加不同浓度 ATA 的 $3 \% \mathrm{NaCl}$ 水溶液中浸泡 $30 \mathrm{~min}$ 后测得的交流阻抗 图. 图形中用阻抗值的高低判别缓蚀性能的好坏, 阻 抗值越大说明其对金属的耐蚀性能越好.

从图 2 可以看出, 所得到的阻抗谱图为不规则

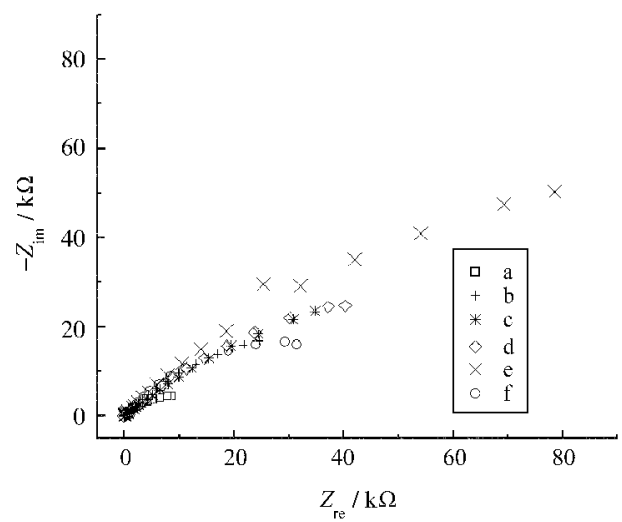

图 2 铜在未加和加入不同浓度缓蚀剂 $\mathrm{ATA}$ 的 $3 \% \mathrm{NaCl}$ 溶液中的 Nyquist 图

Fig.2 Nyquist plots for copper in 3\% $\mathrm{NaCl}$ solution without and with different concentrations of ATA $c_{\text {ATA }} /\left(\mathrm{mmol} \cdot \mathrm{L}^{-1}\right)$ : (a) 0 , (b) 0.06 , (c) 0.12 , (d) 0.18 , (e) 0.24 , (f) 0.30 


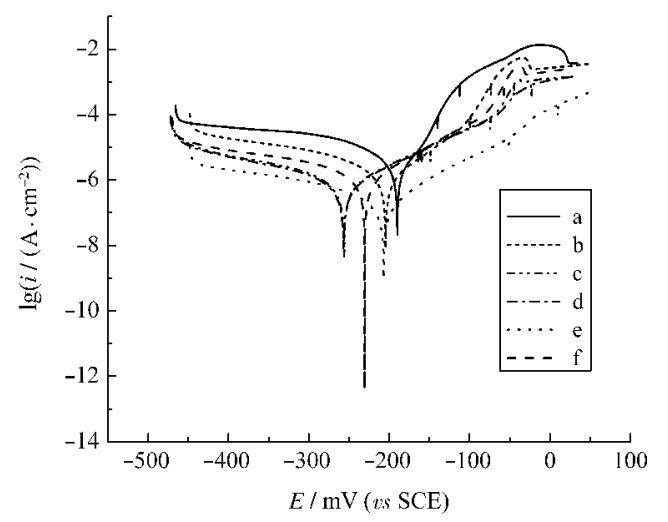

图 3 铜在未加和加入不同浓度缓蚀剂 ATA 的 3\% NaCl 溶液中的极化曲线

Fig.3 Potentiodynamic polarization curve plots for copper in $3 \% \mathrm{NaCl}$ solution without and with different concentrations of ATA

$c_{\text {ATA }} /\left(\mathrm{mmol} \cdot \mathrm{L}^{-1}\right)$ : (a) 0, (b) 0.06 , (c) 0.12 , (d) 0.18 , (e) 0.24 , (f) 0.30

的容抗弧, 表明在所研究体系里铜的腐蚀主要由电 荷传递控制. 弧形与 $Z$ 轴上的弦长对应于铜电极的 电阻 $R_{\mathrm{f}}, R_{\mathrm{f}}$ 越大, 说明缓蚀效果越好 ${ }^{[12]}$. 从图 2 我们 可以看出, 与空白溶液相比, 加人 ATA 缓蚀剂的铜 电极对应的阻抗谱图弦长明显增加, $R_{\mathrm{f}}$ 增大, 说明 缓蚀剂 ATA 对铜腐蚀有明显的缓蚀作用. 另外, 从 图 2 还可以看出, 随着缓蚀剂 ATA 浓度从 0 到 $0.24 \mathrm{mmol} \cdot \mathrm{L}^{-1}$, 对应的阻抗谱图弦长明显增加, $R_{\mathrm{f}}$ 越 大, 缓蚀效果越好, 但 ATA 浓度超过 $0.24 \mathrm{mmol} \cdot \mathrm{L}^{-1}$ 后, 阻抗谱图弦长却呈下降趋势, 因此, 在本实验条 件下, ATA 的最佳浓度为 $0.24 \mathrm{mmol} \cdot \mathrm{L}^{-1}$.

\section{2 不同浓度缓蚀剂下极化曲线的测量}

图 3 为未加和加人不同浓度缓蚀剂 ATA 的 $3 \% \mathrm{NaCl}$ 溶液中铜的极化曲线图. 表 1 列出了由图 3 得出的腐蚀电位 $E_{\mathrm{cor}}$ 腐蚀电流密度 $I_{\mathrm{cor}}$ 和缓蚀率 $\eta$. 缓蚀率 $\eta$ 可按式(1)简便计算, 并以此来衡量该缓 蚀剂在 $3 \% \mathrm{NaCl}$ 溶液中对铜的缓蚀效果:

$$
\eta=\left(\left(I_{\text {corr }}^{0}-I_{\text {corr }}\right) / I_{\text {corr }}^{0}\right) \times 100 \%
$$

表 1 铜在未加和加入不同浓度缓蚀剂 ATA 的 3\% NaCl 溶液中的 $\boldsymbol{E}_{\text {corr }} \boldsymbol{I}_{\text {corr }}$ 和缓蚀率 $\boldsymbol{\eta}$

Table 1 Electrochemical parameters of copper electrodes in $3 \% \mathrm{NaCl}$ solution with different concentration of ATA

\begin{tabular}{cccc}
\hline$c_{\mathrm{ATA}} /\left(\mathrm{mmol} \cdot \mathrm{L}^{-1}\right)$ & $E_{\mathrm{con}} / \mathrm{V}$ & $I_{\mathrm{cor}} /\left(\mu \mathrm{A} \cdot \mathrm{cm}^{-2}\right)$ & $\eta(\%)$ \\
\hline 0.00 & -0.1907 & 15.60 & - \\
0.06 & -0.2048 & 3.006 & 80.73 \\
0.12 & -0.2566 & 0.530 & 96.60 \\
0.18 & -0.2573 & 0.479 & 96.92 \\
0.24 & -0.2075 & 0.366 & 97.65 \\
0.30 & -0.2310 & 0.607 & 92.01 \\
\hline
\end{tabular}

其中 $I_{\mathrm{corr}}^{0}$ 和 $I_{\mathrm{corr}}$ 分别表示未加和加人缓蚀剂 ATA 的 腐蚀电流密度. 如图 3 和表 1 所示, 相对于空白溶 液, 加有缓蚀剂 ATA 的铜电极在 $3 \% \mathrm{NaCl}$ 溶液中 $E_{\text {corr }}$ 明显负移, 说明该缓蚀剂作用的结果是增大了 电极的阴极极化, 抑制了阴极反应过程, 与图 2 结论 一致. 同时 $I_{\mathrm{corr}}$ 明显降低, 缓蚀效率均在 $80 \%$ 以上, 说明 ATA 缓蚀剂对 $3 \% \mathrm{NaCl}$ 溶液中的铜有很好的 缓蚀作用, 其中 ATA 缓蚀剂浓度为 $0.24 \mathrm{mmol} \cdot \mathrm{L}^{-1}$ 时 $I_{\text {corr }}$ 最小, 缓蚀效率达 $97.65 \%$, 缓蚀效果最佳, 这 个结论与交流阻抗得到的结论一致.

\subsection{ATA 缓蚀剂在铜表面的吸附行为}

为研究缓蚀剂 ATA 在铜表面的行为, $25{ }^{\circ} \mathrm{C}$ 下 由极化曲线得到的缓蚀效率 $(\eta)$ 表示其覆盖度 $\theta$. 分 别代人 Temkin、Langmuir、Freundlich 吸附等温式 ${ }^{[13]}$ 拟合. 结果表明 Langmuir 等温式与实验结果符合很 好. 根据 Langmuir 等温式 $[14,15]$ 有

$$
c / \theta=1 / K+c
$$

其中 $c$ 为缓蚀剂浓度, $K$ 为 Langmuir 吸附平衡常数, $\theta$ 为表面覆盖度. 根据表 1 , 将 $c / \theta-c$ 作图 (见图 4), 可 以看出 $c / \theta-c$ 呈很好的线性关系, 线性相关系数 $R$ 为 0.99646 , 斜率为 1.0418 , 说明 ATA 分子在铜表 面的吸附符合 Langmuir 等温式模型, 每一个 ATA 分子大约占据 1.0 个吸附点, 是单分子层吸附. 同时 可得到 Langmuir 吸附平衡常数 $K$ 为 $2.737 \times 10^{5} \mathrm{~L}$. $\mathrm{mol}^{-1}, K$ 与吸附吉布斯自由能 $\Delta G^{0}$ 关系如下 ${ }^{[16]}$ :

$$
K=\frac{1}{55.5} \exp \left(\frac{-\Delta G^{0}}{R T}\right)
$$

其中 $55.5 \mathrm{~mol} \cdot \mathrm{L}^{-1}$ 为溶剂水的浓度, 计算得 $\Delta G^{0}=$ $-40.97 \mathrm{~kJ} \cdot \mathrm{mol}^{-1}, \Delta G^{0}<0$, 说明在等温等压下, ATA

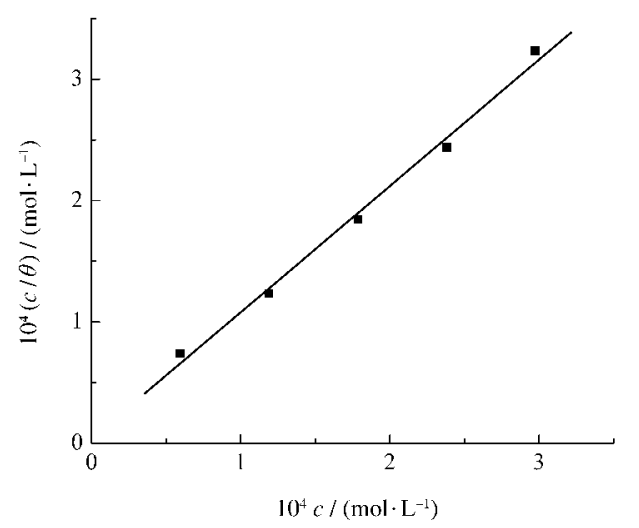

图 4 根据 Langmuir 等温式模型拟合的吸附曲线图

Fig.4 Curve fitting of the corrosion data for copper electrode according to Langmuir thermodynamic kinetic model 


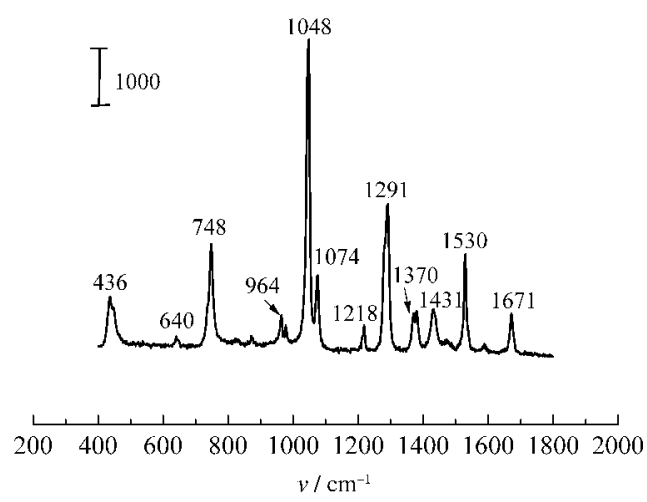

图 5 固体 ATA 的 Raman 光谱

Fig.5 Raman spectrum of solid ATA

分子在铜表面的吸附过程是一种自发行为. 通常情 况下, 当 $\Delta G^{0}$ 在 $-20 \mathrm{~kJ} \cdot \mathrm{mol}^{-1}$ 左右为带电荷的分子 和带电荷的金属之间的静电作用, 为物理吸附; 当 $\Delta G^{0}$ 在 $-40 \mathrm{~kJ} \cdot \mathrm{mol}^{-1}$ 或者更负, 表示带电荷的分子通 过与金属共用电子成键, 为化学吸附 ${ }^{[17,18]}$, 因此, ATA 分子在铜表面的吸附是典型的化学吸附.

\section{4 在 3\% NaCl 溶液中 ATA 对铜电极作用的}

\section{SERS 研究}

图 5 和图 6 分别为 ATA 固体的 Raman 光谱和 在开路电压 $(-0.21 \mathrm{~V})$ 下 ATA 在铜表面的 SERS 光 谱图. 图 7 是 $0.24 \mathrm{mmol} \cdot \mathrm{L}^{-1} \mathrm{ATA}+3 \% \mathrm{NaCl}$ 溶液中 在 $-1.0 \mathrm{~V}$ 到 $0.1 \mathrm{~V}$ 电位范围内 ATA 吸附在铜电极 表面的SERS 谱, 各谱峰的归属见表 $2^{[10,19-23]}$. 得到的 铜表明的光谱图与文献报导类似 ${ }^{[10]}$. 由图 6 可以看 出, 在开路电压下, 741、1053、1216 和 $1287 \mathrm{~cm}^{-1}$ 处 的峰分别归属于三氮唑的环呼吸振动峰, 环面内伸 缩振动峰, 一 $\mathrm{NH}$ 一变形振动峰和一 $\mathrm{CH}$ 面内伸缩振 动峰, 相对于固体 ATA 的 Raman 光谱(图 5), 它们 发生了不同程度的位移; 固体 ATA 的 Raman 光谱 中 $748 、 1048 、 1074 、 1218$ 和 $1291 \mathrm{~cm}^{-1}$ 处的峰分别被

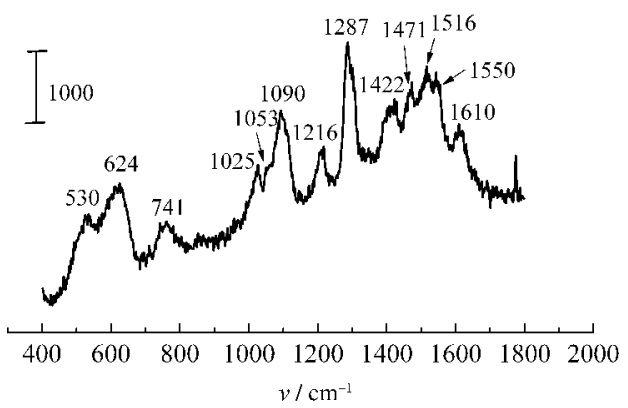

图 6 在开路电压(-0.21 V)下 ATA 在铜表面的 SERS 光谱

Fig.6 SERS spectrum of ATA adsorbed onto copper at open circuit potential $(-0.21 \mathrm{~V})$

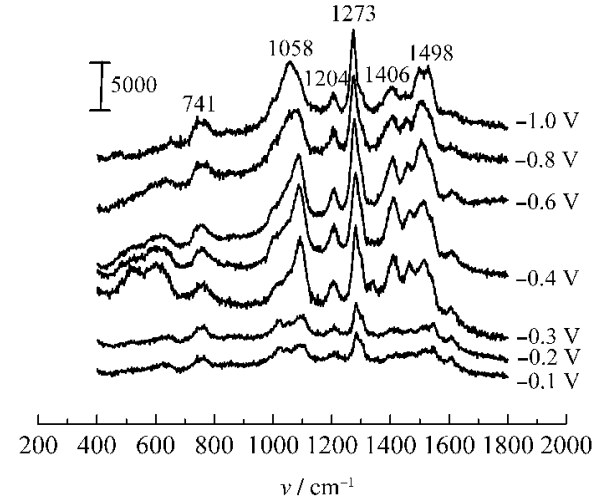

图 7 不同电位下 $0.24 \mathrm{mmol} \cdot \mathrm{L}^{-1} \mathrm{ATA}$ 在铜表面上的 SERS 光谱

Fig.7 SERS spectra of $0.24 \mathrm{mmol} \cdot \mathrm{L}^{-1}$ ATA on copper at different potentials

表 2 固体 ATA 的拉曼光谱和 3\% NaCI 溶液中 ATA 吸 附在铜电极表面的 SERS 谱峰指认

Table 2 Assignment of Raman spectrum of solid ATA and SERS of ATA on $\mathrm{Cu}$ in $3 \% \mathrm{NaCl}$ solution

\begin{tabular}{clcc}
\hline \multirow{2}{*}{ Solid ATA } & \multicolumn{1}{c}{ Assignment } & $-0.21^{*}$ & -0.6 \\
\cline { 2 - 4 } & & $741(\mathrm{w})$ & $752(\mathrm{w})$ \\
$748(\mathrm{~s})$ & triazole ring breathing & - & $1080(\mathrm{~m})$ \\
$1048(\mathrm{vs})$ & in plane triazole ring stretching & $1053(\mathrm{w})$ & - \\
$1074(\mathrm{~m})$ & $-\mathrm{N}-\mathrm{N}-$ stretching & $1216(\mathrm{w})$ & $1209(\mathrm{w})$ \\
$1218(\mathrm{w})$ & $-\mathrm{NH}-$ deformation & $1287(\mathrm{vs})$ & $1286(\mathrm{~s})$ \\
$1291(\mathrm{~s})$ & $-\mathrm{CH}$ in plane bending &
\end{tabular}

指认为环呼吸振动峰, 环面内伸缩振动峰, 一 $\mathrm{N}-$ $\mathrm{N}$ 一振动峰, 一 $\mathrm{NH}$ 一变形振动峰和一 $\mathrm{CH}$ 面内伸缩 振动峰, 在不同电位下 ATA 的吸附会有所不同, 相 应的拉曼强度也不同(见图 7), 当电位为 $-0.6 \mathrm{~V}$ 时, 谱峰强度最大. 表 2 中仅列出电位为 $-0.6 \mathrm{~V}$ 时某些 特征谱峰的强度. SERS 分析表明, 在 $3 \% \mathrm{NaCl}$ 溶液 中, ATA 已与铜发生作用, 铜表面特征峰的存在表 明, 铜表面形成了一种含有 ATA 或与铜形成配合 物的膜. ATA 分子吸附于铜表面达到抑制金属腐蚀 的作用, 是 $\mathrm{ATA}^{-}$与 $\mathrm{Cu}^{+}$形成配合物来阻止氯离子化 合物 $\left(\mathrm{CuCl}_{2}^{-}\right)$的生成.

\section{3 结 论}

(1) ATA 对铜在 $3 \% \mathrm{NaCl}$ 溶液中有较好的缓蚀 效果, 缓蚀剂浓度为 $0.24 \mathrm{mmol} \cdot \mathrm{L}^{-1}$ 时, ATA 的缓蚀 效果最佳, 缓蚀效率达 $97.65 \%$.

(2) 等温等压条件下, ATA 能自发地吸附在铜 表面. ATA 在 $3 \% \mathrm{NaCl}$ 溶液中对铜表面的吸附服从 Langmuir 等温式, 是典型的化学吸附.

(3) 在 $3 \% \mathrm{NaCl}$ 溶液中, ATA 的 SERS 图表明 
ATA 分子通过很强的吸附于铜表面达到抑制其腐 蚀的作用, 是与 $\mathrm{Cu}^{+}$形成配合物来阻止氯离子化合 物 $\left(\mathrm{CuCl}_{2}^{-}\right)$的生成.

\section{References}

1 Xu, Q. J.; Wan, Z. Y.; Yin, R. H.; Zhu, L. J.; Cao, W. M.; Zhou, G D.; Lin, C. J. Acta Chim. Sin., 2007, 6(18): 1981 [徐群杰, 万宗 跃, 印仁和, 朱律均, 曹为民, 周国定, 林昌健. 化学学报, 2007, 65(18): 1981]

2 Zhu, L. J.; Xu, Q. J.; Cao, W. M.; Wan, Z. Y.; Zhou, G. D.; Lin, C. J. Acta Phys. -Chim. Sin., 2008, 24(5): 805 [朱律均, 徐群杰, 曹 为民, 万宗跃, 周国定, 林昌健. 物理化学学报, 2008, 24(5): 805]

3 Xu, Q. J.; Zhou, G. D.; Lu, Z.; Tian, Z. Q.; Lin, C. J. Chin. J. Appl. Chem., 2002, 19(4): 390 [徐群杰, 周国定, 陆 柱, 田中群, 林 昌健. 应用化学, 2002, 19(4): 390]

4 Xu, Q. J.; Zhou, G. D.; Lu, Z.; Tian, Z. Q.; Lin, C. J. Acta Chim. Sin., 2001, 59(6): 950 [徐群杰, 周国定, 陆 柱, 田中群, 林昌 健. 化学学报, 2001, 59(6): 950]

5 Xu, Q. J.; Zhou, G. D.; Lu, Z.; Yang, Y.; You, J. K.; Lin, C. J. Acta Chim. Sin., 2000, 58(9): 1079 [徐群杰, 周国定, 陆 柱, 杨 勇, 尤金跨, 林昌健. 化学学报, 2000, 58(9): 1079]

6 Zhang, D. Q.; Gao, L. X.; Zhou, G. D.; Kang, Y. L. Appl. Electrochem., 2008, 38: 71

7 Elmorsi, M. A.; Hassanein, A. M. Corrosion Science, 1999, 41: 2337

8 Deyab, M. A.; Abo Dief, H. A.; Eissa, E. A.; Tamn, A. R. Electrochim. Acta, 2007, 52: 8105

9 Lalitha, A.; Ramesh, S.; Rajeswari, S. Electrochim. Acta, 2005, 51: 47

10 El-Sayed, M. S.; Erasmus, R. M.; Comins, J. D. Colloid and
Interface Science, 2007, 311: 144

11 Wafaa, Q.; Christine, B.; Nadine, P.; Hisasi, T.; Abellah, S.; Georges, M. Electrochim. Acta, 2002, 47: 4339

12 Xu, Q. J.; Zhou, G. D.; Wang, H. F.; Cai, W. B. Electrochemistry, 2006, 12(1): 65 [徐群杰, 周国定, 王会峰, 蔡文斌. 电化学, 2006, 12(1): 65]

13 Cao, C. N. Principles of corrosive electrochemistry. 2nd ed. Beijing: Chemical Industry Press, 2004: 246-250 [曹楚南. 腐蚀 电化学原理. 第二版. 北京: 化学工业出版社, 2004: 246-250]

14 Wang, X. Q.; Liu, R. Q.; Zhu, L. Q.; Gong, J. W. Acta Phys. -Chim Sin., 2007, 23(1): 21 [王献群, 刘瑞泉, 朱丽琴, 宫建伟. 物理化 学学报, 2007, 23(1): 21]

15 Oguzie, E. E.; Li, Y.; Wang, F. H. Colloid and Interface Science, 2007, 310: 90

16 Moussa, M. N. H.; El-Far, A. A.; El-Shafei, A. A. Materials Chemistry and Physics, 2007, 105: 105

17 Villamil, R. F. V.; Corio, P.; Rubim, J. C.; Agostinho, S. M. L. J. Electroanal. Chem., 2002, 535: 75

18 Hong, H. G.; Wonchoul, P. Electrochim. Acta, 2005, 51: 579

19 Wei, W. J.; Fu, H. T.; Li, Y.; Zhu, Y. F. Acta Phys. -Chim. Sin., 2002, 18(2): 152 [魏无际, 付海涛, 李 瑛, 朱一帆. 物理化学 学报, 2002, 18(2): 152]

20 Gu, R. A.; Bao, F.; Shen, X. Y.; Cui, Y.; Yao, J. L. Chem. J. Chin. Univ., 2007, 28(5): 948 [顾仁敖, 鲍 芳, 沈晓英, 崔 颜, 姚建 林. 高等学校化学学报, 2007, 28(5): 948]

21 Huynh, N.; Bottle, S. E.; Notoya, T.; Schweinsberg, D. P. Corrosion Science, 2002, 44: 2583

22 Otieno-Alegoa, V.; Huynh, N.; Notoya, T.; Bottle, S. E.; Schweinsberg, D. P. Corrosion Science, 1999, 41: 685

23 Cao, P. G.; Yao, J. L.; Zheng, J. W.; Gu, R. A.; Tian, Z. Q. Langmuir, 2002, 18: 100 